\title{
A statistical method for draft tube pressure pulsation analysis
}

\author{
P K Doerfler and N Ruchonnet \\ Andritz Hydro Ltd, R\&D/EngM, Hardstrasse 319, 8021 Zurich, Switzerland \\ E-mail: peter.doerfler@andritz.com
}

\begin{abstract}
Draft tube pressure pulsation (DTPP) in Francis turbines is composed of various components originating from different physical phenomena. These components may be separated because they differ by their spatial relationships and by their propagation mechanism. The first step for such an analysis was to distinguish between so-called synchronous and asynchronous pulsations; only approximately periodic phenomena could be described in this manner. However, less regular pulsations are always present, and these become important when turbines have to operate in the far off-design range, in particular at very low load. The statistical method described here permits to separate the stochastic (random) component from the two traditional 'regular' components. It works in connection with the standard technique of model testing with several pressure signals measured in draft tube cone. The difference between the individual signals and the averaged pressure signal, together with the coherence between the individual pressure signals is used for analysis. An example reveals that a generalized, non-periodic version of the asynchronous pulsation is important at low load.
\end{abstract}

\section{Introduction}

\subsection{Synchronous and asynchronous pulsations}

1.1.1. Early studies. In the flow downstream of the runner in a reaction turbine, pressure pulsations are often a matter of concern because they may produce mechanical vibration, power swings and other undesirable effects. In the very large body of literature on the subject, the majority of studies is concerned with the draft tube pressure pulsations (DTPP) of Francis turbines, and in particular with a type of pulsation occurring at partial load. In laboratory tests using a reduced-scale model turbine, it is easily recognized due to the characteristic corkscrew shape of the cavitating vortex downstream of the runner. It is well-known that this pulsation is composed of two different phenomena occurring simultaneously with the same frequency. The distribution of amplitudes and phase of pressure fluctuations on the surface of the draft tube cone can only be explained reasonably if a distinction between a so-called asynchronous and a synchronous pulsation is made. The terms 'synchronous' and 'asynchronous' refer to the behavior of the phase around the circumference of the draft tube, and not to the frequency of the phenomenon that is usually a small fraction of the runner's rotational speed. Nishi et al [1] introduced this distinction and devised an analytical method how to separate the two components. Doerfler [2] showed that the concept permits to quantitatively describe the influence of vortex cavitation on the DTPP amplitudes, including their differences around the draft tube circumference. 
1.1.2. Definitions. The synchronous component has equal phase and amplitude for all locations in the same cross section; it may be understood as a plane pressure wave propagating through the draft tube and also through the rest of the water conduit, though there may be a radial gradient in the draft tube itself. The asynchronous component is a pressure pattern rotating about the circumference of the draft tube; its period corresponds to the circumference, and it may have harmonics though the fundamental component is the most important one. Both components are produced by the movement of the corkscrew vortex; their frequency is the same namely, the precession frequency of the corkscrew vortex. In other near-periodic pulsations, for instance at high load, the asynchronous pulsation may be missing; on the other hand in rotationally symmetric draft tubes there is no synchronous component, as Nishi et al [1] showed.

Only few applications of the concept may be found in the literature; but the distinction between the asynchronous and synchronous component has practical value because their mechanical effects are different.

\subsection{Load influence on pulsation regimes in a Francis turbine}

1.2.1. Model data. The data for the practical examples in this paper have been measured on a model Francis turbine with a specific speed of $\mathrm{n}_{\mathrm{QE}}=0.20$. The reference (runner exit) diameter of the model is $\mathrm{D}_{2}=0.352 \mathrm{~m}$. The tests were part of a commercial project; accordingly the standard arrangement of pressure sensors in the spiral casing and draft tube was used. If not specified otherwise, there are normally four pressure transducers located in the same cross section of the draft tube cone, with an equal $90^{\circ}$ circumferential spacing. Some additional sensors (e.g. in the spiral casing entry) will not be discussed here.
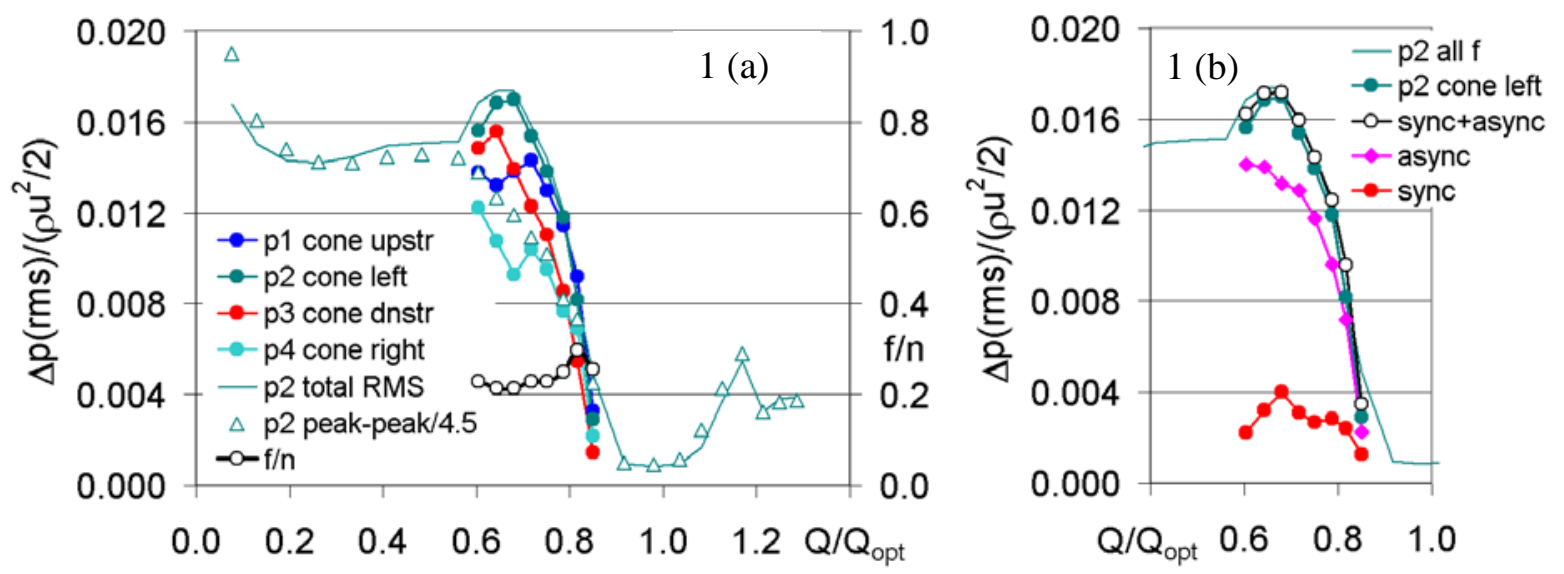

Figure 1. Results of conventional pulsation analysis

1.2.2. Test series, recording and analysis data. In a test series corresponding to constant speed factor $n_{E D}=0.444$ and with cavitation number $\sigma=0.36$, pressure pulsation was measured for 25 values of discharge, starting from speed-no load condition. The frequency of rotation was $n=19.16 \mathrm{~Hz}$; a $40 \mathrm{~s}$ record with a sampling frequency $f_{s}=500 \mathrm{~Hz}$ was sampled for every test point; the low-pass filtering permitted evaluation up to $230 \mathrm{~Hz} \cong 12 \mathrm{n}$. Multi-channel analysis was performed with a frequency resolution $\Delta f=25 \mathrm{mHz}$, or $1.3 \%$ of $n$. This corresponds to a sample size of $N=2 f_{s} / \Delta f=2000$ points. The results for the four draft tube cone pressures, measured $D_{2} / 2$ below the guide vane center line,are discussed in this paper. In this turbine, the standard evaluation for synchronous and asynchronous pulsation with approximately periodic precession of the vortex rope was present in the load range between 60 and 85 percent of $Q_{n D, o p t}$, as may be seen in figure 1(a). 
1.2.3. Results of conventional pulsation analysis. In the figure 1 (a) the intensity of the 4 draft tube pressure signals is shown as rms value of a narrow-band frequency interval centered at the precession frequency. Using these values, and the relative phase angles, the conventional analysis was performed and figure 1(b) shows the resulting components. Because of the rather high $\sigma$ value, the synchronous component is quite small compared to the asynchronous component. The scalar sum of the two components is also shown; for every load condition, this scalar sum is the maximum possible amplitude in some point at the circumference, and it happens to occur close to the sensor p2 (right side of cone), as seen from the comparison in figure 1(b). In addition to the narrow-band rms values, the left-hand graph also shows the wide-band rms comprising all frequencies lower than $3 \mathrm{n}$. For the analyzed range, the vortex frequency obviously makes up for most of the pulsation. The values outside of this range show that there are large pulsations which cannot be analyzed by this method, yet may be interesting too.

\section{Principle of statistical analysis}

\subsection{Averaged signal and spectrum}

For a normal model test for pressure pulsation, the pertinent international standard, IEC 60193 [3] recommends using at least 2, but preferably more sensors in a plane perpendicular to the axis of draft tube cone. Very often 4 sensors are used, as in our experiment. For a test point with $Q / Q_{o p t}=0.56$, a short time series of the four signals is shown in figure 2. The signals are numbered in the sense of runner rotation, starting on the upstream side of the draft tube.
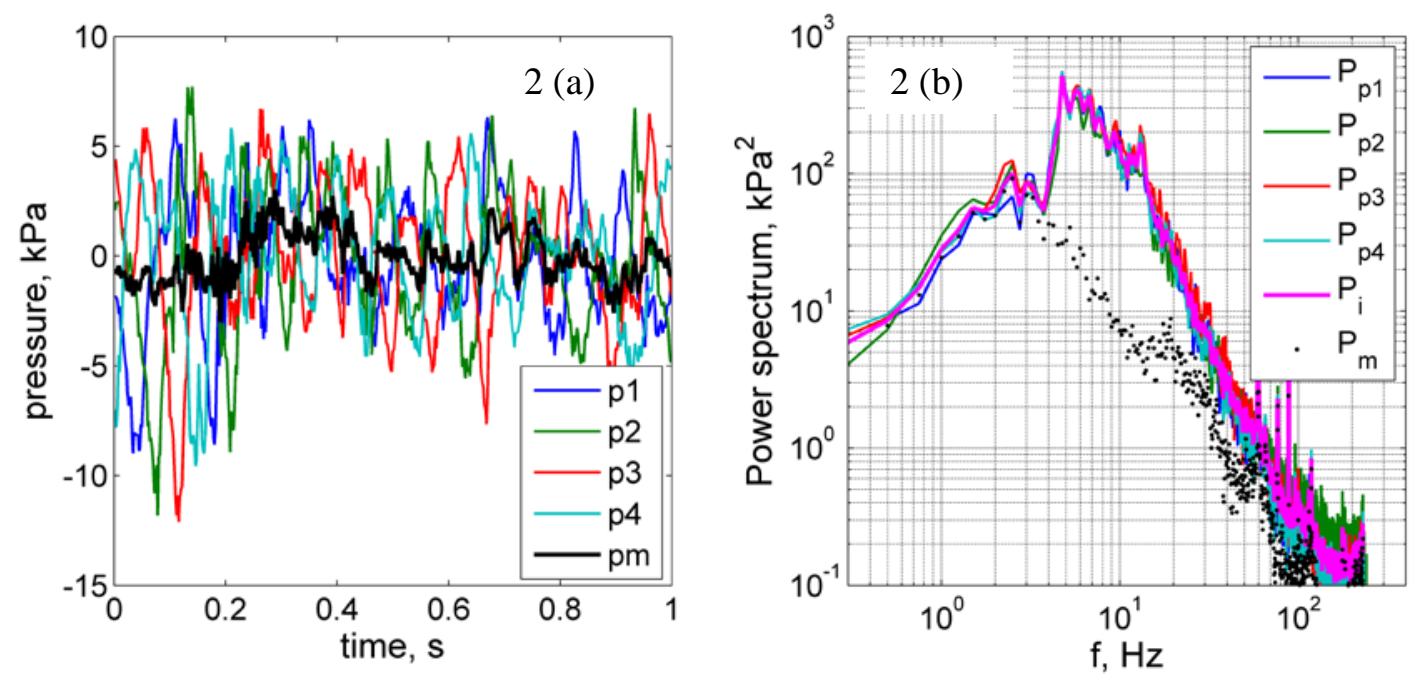

Figure 2. Time domain signals (left) and power spectra (right)

We may create an averaged signal $\mathrm{p}_{\mathrm{m}}(\mathrm{t})$ for the whole sampled time series

$$
p_{m}=\left(p_{1}+p_{2}+p_{3}+p_{4}\right) / 4
$$

and treat it in the same manner as the original signals, in particular, we may compute its power spectrum $\mathrm{P}_{\mathrm{m}}(\mathrm{f})$, and its phase and coherence with the other signals. Also, we may compute an averaged power spectrum

$$
p_{i}=\left(p_{p 1}+p_{p 2}+p_{p 3}+p_{p 4}\right) / 4
$$

based on the individual frequency spectra of the 4 signals. If we compare the spectra, as in the righthand graph in figure 2, we note an interesting property of the spectrum for $\mathrm{p}_{\mathrm{m}}$ : it is always smaller than the averaged spectrum of the $\mathrm{p}_{\mathrm{i}}$. This is not really surprising; the reason is that not only the asynchronous pulsation but, due to the law of large numbers, also most of the random pulsation cancels out in averaging for $\mathrm{p}_{\mathrm{m}}$. This effect helps to estimate which fraction $\mathrm{P}_{\mathrm{r}}$ of the spectrum pertains 
to the random fluctuation. If we think of the pulsation characterized by $\mathrm{P}_{\mathrm{i}}$ being composed of a synchronous, an asynchronous, and a random component, with spectra $\mathrm{P}_{\mathrm{s}}, \mathrm{P}_{\mathrm{a}}$ and $\mathrm{P}_{\mathrm{r}}$,

$$
p_{s}+p_{a}+p_{r}=p_{i}
$$

then we may also assume the asynchronous component as being cancelled out in the averaged signal, and the random spectrum being reduced by the number of channels averaged

$$
p_{s}+p_{r} / 4=p_{m}
$$

\subsection{Coherence between pressure signals}

From multi-channel analysis of the pressure signals, we may obtain not only the phase relationships but also the coherence between the signals. The coherence may be taken as a measure for signal-tonoise ratio, and thus be used to sort out the random components. Using the non-synchronous portion

$$
p_{i}^{\prime}=p_{i}-p_{m}, i=1,2,3,4
$$

instead of the signals $p_{i}$, the coherence $\gamma$ between neighbouring signals $p_{i}$ ' and $p_{i+1}$ ' may be used for estimating the ratio between the random and asynchronous component:

$$
(\gamma-1) \cdot P_{a}+\gamma \cdot P_{r}=0
$$
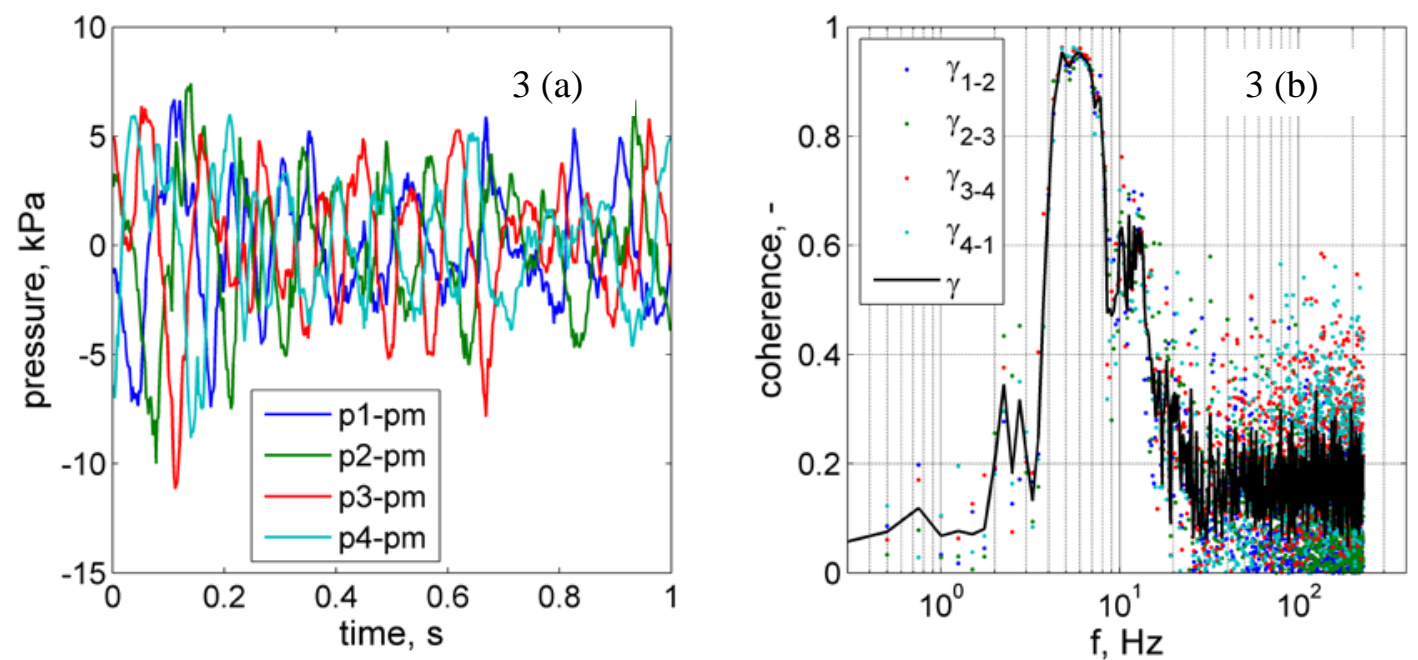

Figure 3. Non-synchronous signals $\mathrm{p}_{\mathrm{i}}$ ' (left) and their coherence (right)

\subsection{Power spectra and linear spectra of components}

The system composed of equations (3), (4) and (6) can be solved for all frequencies $f$ to obtain the power spectra $P_{s}, P_{a}$ and $P_{r}$ of the three components. From the power spectra $P_{x}$ one may derive linear spectra $S_{x}$ using

$$
S_{x}=S_{X}=\sqrt{\left(2 \Delta f \cdot P_{X} / f_{s}\right)}=\sqrt{\left(2 P_{X} / N\right)}
$$

With this definition, every value of $S_{x}(f)$ is the rms value of the oscillation pertaining to the frequency band $f \pm \Delta f / 2$. The overall rms intensity $\sqrt{ } \Sigma\left(S_{x}^{2}\right)$ with summation over all frequencies is the standard deviation of the signal $p_{x}$.

\section{Application to the test case}

\subsection{Load influence on pulsation types}

The spectra $S_{x}$ of the three components have been evaluated in every point of the test case. Each spectrum was integrated to obtain the total intensity; its distribution versus load is shown in figure 4 . 
This is in analogy to figure 1, but now the stochastic component $S_{r}$ is quantified, and a different procedure has been used to determine the asynchronous pulsation. In both figures, the rms values are made non-dimensional by dividing though the dynamic pressure $\rho u^{2} / 2$ based on $u=D_{2} \pi$. In addition to the pressure spectra, a measure for the hydraulic losses is also shown in figure 4 , using the left-hand scale. The ratio $\left(Q_{E D}-P_{E D}\right) / Q_{E D o p t}$ represents the hydraulic losses per unit time.

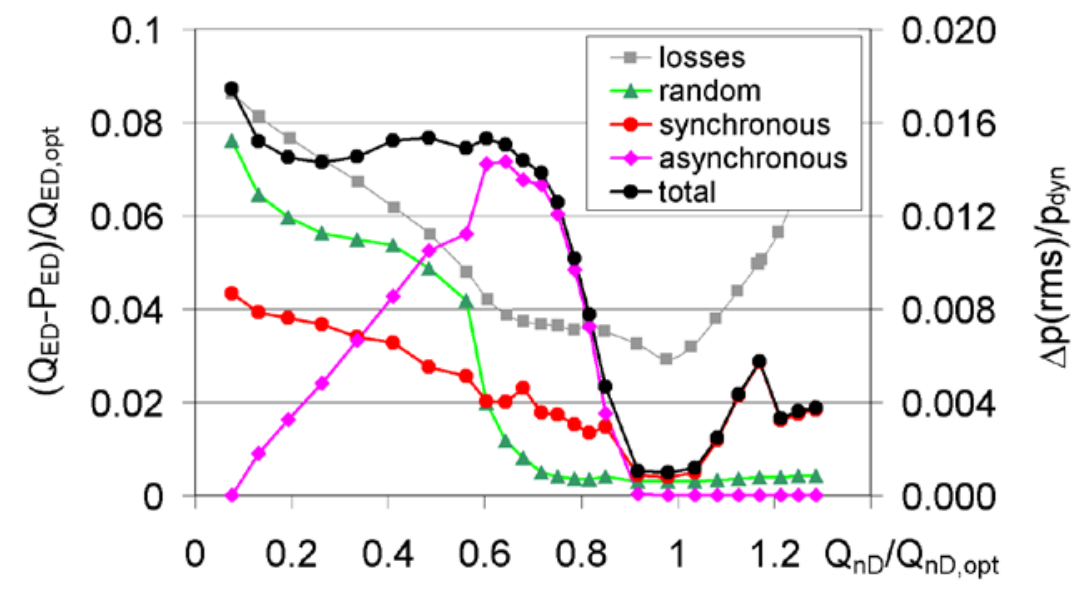

Figure 4. Distribution of pulsation components (right scale) and hydraulic loss

3.1.1. Partial load. The condition shown as log-log spectra in figure 5(a) corresponds to the flow regime of the well-known part-load vortex. The intensity of the synchronous and asynchronous components is reproduced with small deviations from figure 1. The stochastic component (green curve) is small and its spectrum is smooth and its high-frequency part resembles a power law which is typical for turbulence. Most of the spectral intensity above $30 \mathrm{~Hz}(1.5 \mathrm{n})$ is identified as synchronous pulsation.

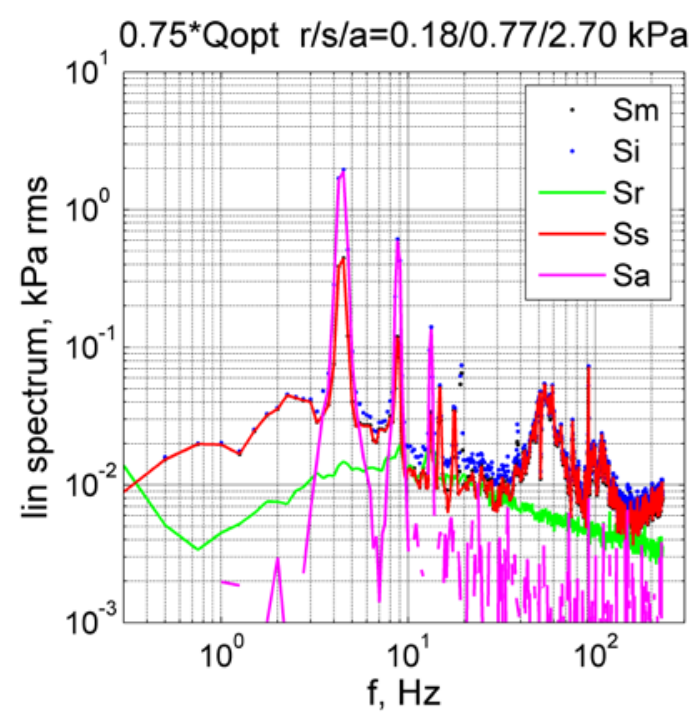

Figure 5. (a) Spectra from partial load

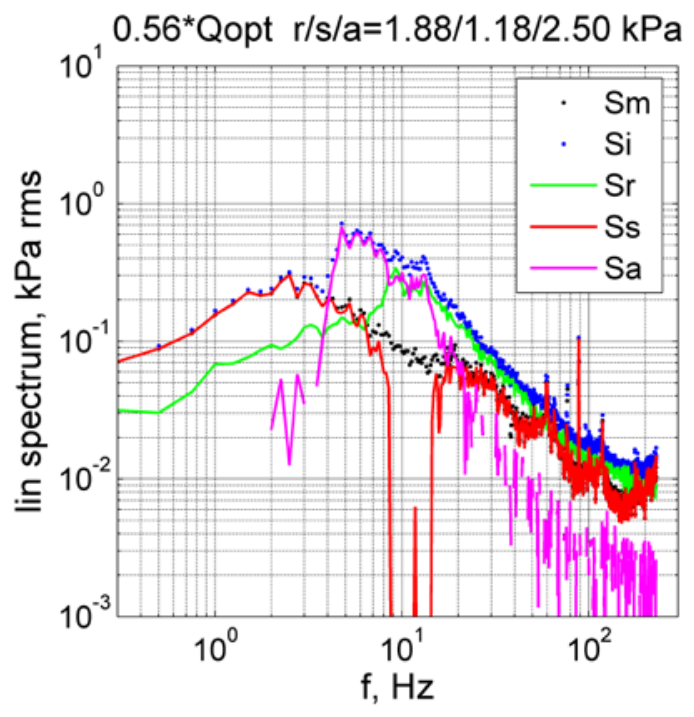

Figure 5. (b) Spectra from low load

3.1.2. Low load. As shown in figure 1, the regular part-load vortex disintegrates in this turbine when the discharge drops below $60 \%$ of $Q_{\text {opt }}$. The point shown in figure 5(b) was measured just below this limit, at $Q / Q_{o p t}=0.56$. The diagram shows that the pulsation is not purely stochastic but has important asynchronous components which are, however, distributed over a wider range of frequencies. Despite the fact that the conventional method cannot describe this flow, it is obvious that less regular vortex 
structures persist; this can be seen from the coherence between the non-synchronous signals in figure 6 (a) and from the averaged phase relationships shown in figure 6(b). Despite of the less periodic nature of the fluctuations in the lower-load points (red and blue coloured), vortex structures with a precession can still be detected by means of their phase shifts of $\pi / 2$ and multiples thereof (figure 6 (b)).

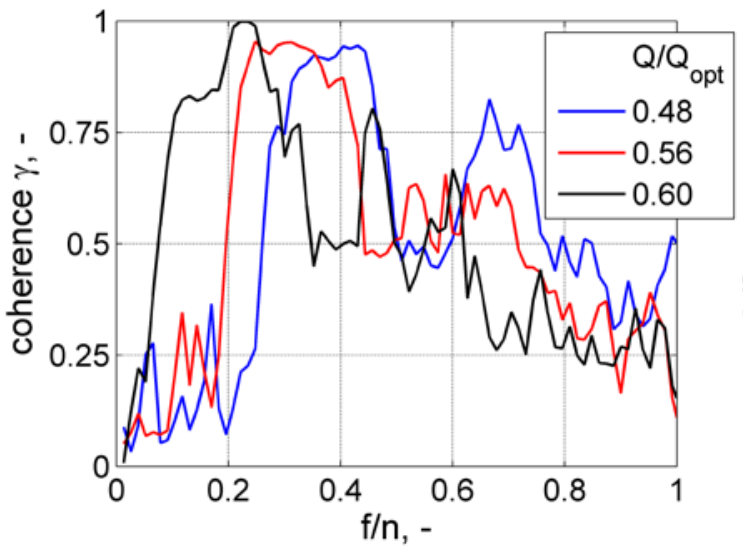

Figure 6. (a) Coherence of asynchronous parts

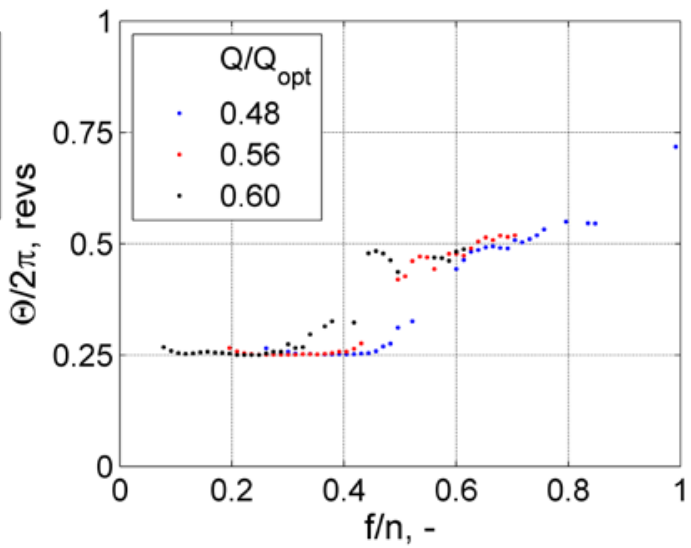

Figure 6. (b) Phase difference between asynchronous parts, $\chi(f)>0.5$ only

3.1.3. Very low load. At very low load (speed-no load, figure 7(a)), almost no asynchronous pulsation is detected; some synchronous pulsation is the only coherent component. This is the final stage of the decay of coherent vortex structures that starts when the lower limit of the single-helix region is surpassed.

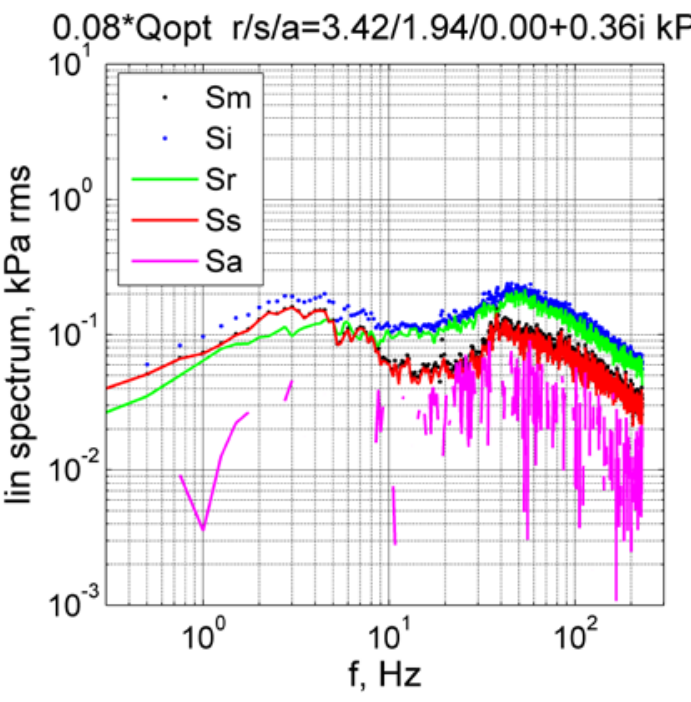

Figure 7. (a) Spectra from speed-no load

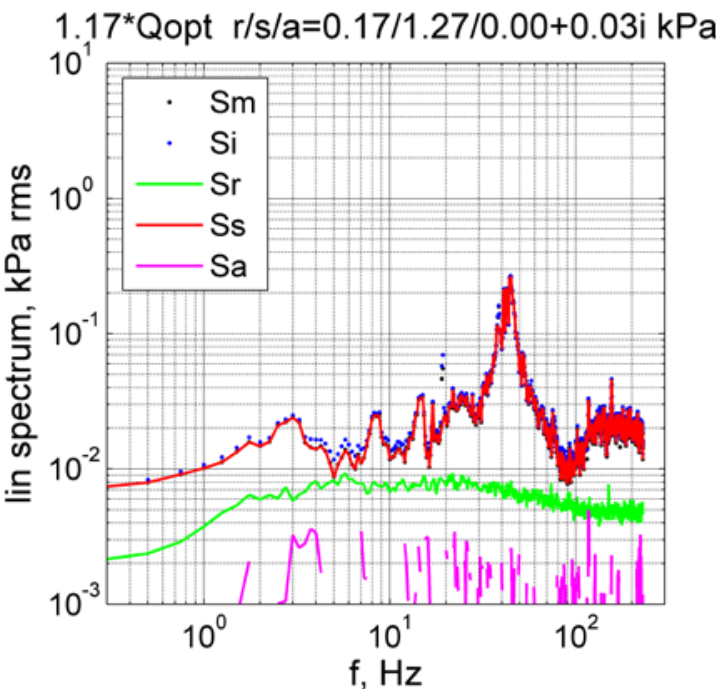

Figure 7. (b) Spectra from high load

3.1.4. High load. No asynchronous pulsation occurs in the range where the discharge exceeds the best-efficiency value, see figure 7(b). The random pulsation remains very low and almost all of the pulsation in the draft tube cone is synchronous. This test point shows that the procedure for separating the stochastic component from the much higher synchronous pulsation works remarkably well.

\subsection{Other findings}


3.2.1. Characteristic of random component. All the random spectra resulting from the analysis are very smooth and their high-frequency parts may be approximated by a power law whose exponent depends somewhat on the test condition. For the low-load points, these random spectra are represented in the figure 8 . The last three cases in figure 8 are already inside the regular part-load vortex zone.

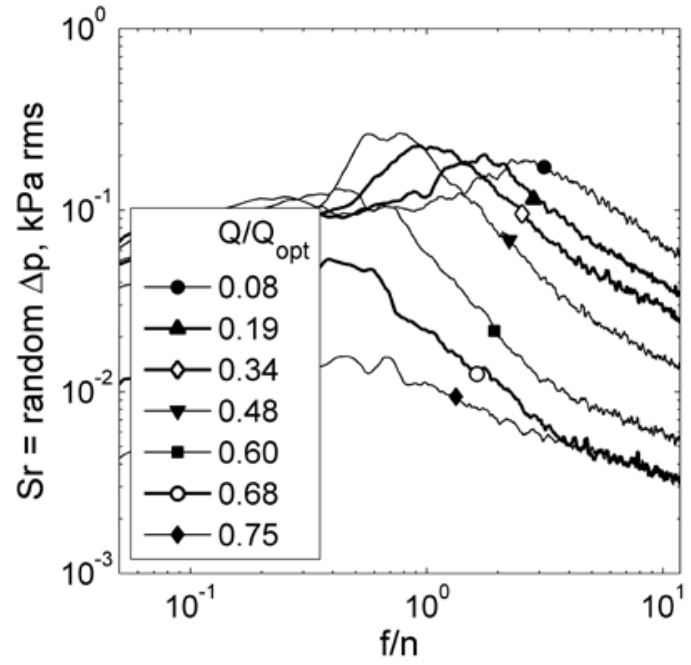

Figure 8. Spectra of the stochastic pulsation

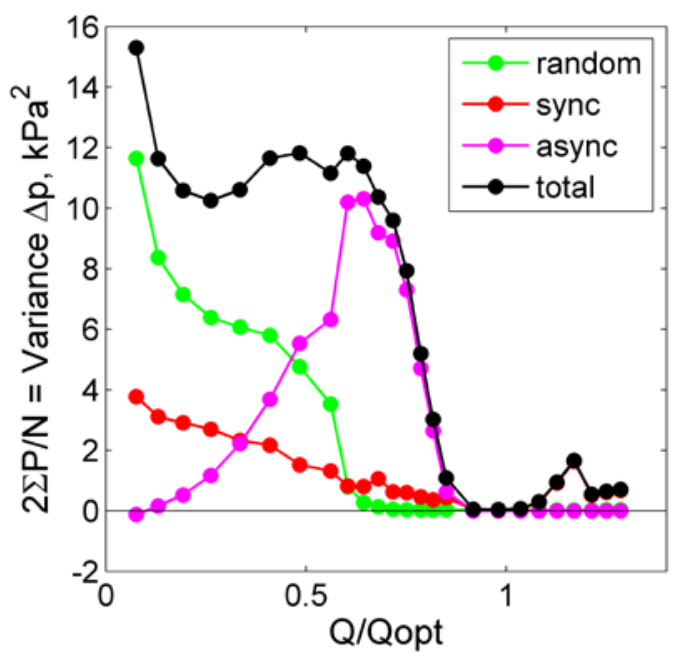

Figure 9. Total spectral power of components

3.2.2. Dropout of asynchronous or synchronous spectrum. In some cases, e.g. the tests shown in figures 5(b) and 7(a), a portion of the frequency range may erroneously result with a negative power spectrum. The 'empty' portions of the asynchronous spectra pertain to frequencies where the estimated $\mathrm{P}_{\mathrm{a}}$ is negative and the square root in equation (7) becomes imaginary. The same may also occur occasionally with parts of the synchronous spectrum, as in figure 5(b). For the total intensity of each spectral component, these errors play a negligible role. Actually, there are large portions of the spectra where asynchronous pulsations are absent, and the estimate for $P_{a}(f)$ is close to zero. In a some points the also overall intensity of the asynchronous power spectrum becomes negative. The inaccuracy is very small, as shown by the small negative overshoot of the estimated power spectrum on the lower and higher end of the 'async' curve in figure 9; it simply indicates that there is no asynchronous component in this load range.

Apart from statistical estimation errors, there may also be other reasons for some of these deviations. Maybe the most important one is that, due to the draft tube elbow, the problem is not rotationally symmetric, but this is implicitly assumed by the averaging procedures in equations (1) and (2).

3.2.3. Parasitic components. Foreign components of the measured signal which belong to none of the three categories show up in case of low total intensity, near $Q=Q_{o p t}$. The frequency of rotation and its harmonics make up for the major part of the disturbances. Due to this fact, it was possible to eliminate most of the disturbance from the spectra; its overall intensity (about $0.1 \mathrm{kPa} \mathrm{rms}$ ) is very small compared to the useful signal.

3.2.4. High partial load. Like in many other Francis models, a typical kind of pulsation occurs in this model in the range between $0.72<Q / Q o p t<0.85$. In the high-partial load pulsation, or " $80 \%$ pulsation", occurring here with frequencies of $1.5 \div 7$ times the runner frequency, there are parts with poor coherence that seem to be not clearly synchronous or asynchronous. In the evaluation of some points, it caused a maybe unjustified increase of the random component in the frequency range concerned. 
This deviation is too small to appear in the overall intensity but it would be desirable to find some way to clarify or avoid the bias.

\section{Concluding remarks}

\subsection{Possible use}

The method described in this paper is a more general alternative for Nishi's method; it is able to describe also non-periodic phenomena. This ability will be valuable for assessing and comparing different turbine models in the far off-design range. In this range there is less data available so far, compared to the usually guaranteed range of operation. However, operation in the low-load range is becoming more frequent and, at the same time, it plays an important role with respect to fatigue of components including the runner. It appears possible, after some experience with the new type of analysis, to establish a correlation between the pulsations in the draft tube cone and the kind of dynamic loads on the runner that is not related to rotor-stator interaction.

\subsection{Statistical principle}

Equation (6) is an extremely simple approach to separate the two non-synchronous components. Its main justification is in the plausibility of results. Apart from that, some connection between the coherence and the signal-to-noise ratio should certainly exist - here the asynchronous pulsation is interpreted as signal and the stochastic pulsation as noise [4]. A statistically sound approach would probably be very complicated.

\section{References}

[1] Nishi M, Matsunaga S, Kubota S and Senoo Y Surging characteristics of conical and elbowtype draft tubes IAHR Section Hydraulic Machinery, Equipment and Cavitation, 12th Symp. (Stirling, UK, 1984)

[2] Doerfler P K System dynamics of the Francis turbine half-load surge IAHR Section Hydraulic Machinery, Equipment and Cavitation, 11th Symp. (Amsterdam, Netherlands, 1982)

[3] IEC standard 60193, ed. 2.01999 Hydraulic turbines, storage pumps and pump-turbines Model acceptance tests

[4] Bendat J S and Piersol A G 2010 Random Data: Analysis and Measurement Procedures (USA: Wiley) 